\title{
How to implement the new 2018 ESH/ESC guidelines for the management of arterial hypertension in patients with metabolic syndrome and diabetes?
}

\author{
Krystyna Widecka, Michał Janowski \\ Department of Hypertension and Internal Medicine, Pomeranian Medical University, Szczecin, Poland
}

\begin{abstract}
Blood pressure (BP) control in hypertensive patients remains poor worldwide, particularly in high-risk patients with hypertension and diabetes.

New ESH/ESC guidelines recommend more stringent BP targets (on-treatment values of $\leq 130 / 80 \mathrm{~mm} \mathrm{Hg}$ in the general population and $\leq 140 / 90 \mathrm{~mm} \mathrm{Hg}$ in older hypertensive people), which will make the achievement of BP control even more challenging.

The most effective evidence-based treatment strategy to improve BP control is one that: encourages the use of combination treatment, enables the use of single-pill combination (SPC) therapy for most patients to improve adherence to treatment, with the use of SPC therapy as initial therapy.

The combination of perindopril with thiazide-like diuretic indapamide is one of the widely tested SPC in multicentre clinical trials for diabetic patients. Studies suggested that two-drug combination therapy will control BP in approximately two-thirds of patients. For patients whose BP is not controlled by two-drug combination therapy, the logical option is to increase treatment to three-drug combination therapy: usually a RAS blocker, a CCB and a diuretic.

The combination of perindopril with indapamide and amlodipine is particularly recommended in population of hypertensive patients with metabolic complications because of favourable, neutral effect on blood glucose and cholesterol level. This combination has proven hypotensive effect in group of patients with difficult-to-control hypertension.
\end{abstract}

Key words: guidelines ESH/ESC 2018; hypertension; diabetes, single-pill combination

Arterial Hypertens. 2018, vol. 22, no. 4, pages: 156-162

DOI: 10.5603/AH.a2018.0022

\section{A new strategy for the treatment of hypertension}

Hypertension is currently the most important mortality risk factor in the world and a major public health problem, especially in developed countries. It is esti- mated that currently there are over one billion people living with hypertension worldwide, and by 2025 this number is predicted to increase by $60 \%[1,2]$.

Thanks to the advances in antihypertensive drug therapy we can effectively lower blood pressure in

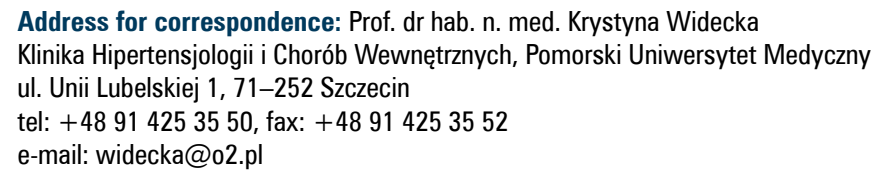


individual patients and, thereby, reduce the main risk factor of cardiovascular (CV) events. Despite this, epidemiological studies clearly indicate poor blood pressure control in the whole population. The lowest rate of adequate control of hypertension is observed among patients with diabetes, chronic kidney disease, stable angina, acute coronary syndrome and left ventricular dysfunction [3-5].

The authors of the 2018 European Society of Hypertension/European Society of Cardiology (ESH/ /ESC) guidelines emphasize that their main goal was to develop a strategy that ensures improved blood pressure (BP) control in treated patients. The most important elements of this strategy are:

\section{Lowering of blood pressure treatment targets}

According to current guidelines, BP should be lowered to less than $140 / 90 \mathrm{~mm} \mathrm{Hg}$ in all patients and if the treatment is well tolerated, further lowering of $\mathrm{BP}$ to $130 / 80 \mathrm{~mm} \mathrm{Hg}$ or less, but not below $120 / 70$ $\mathrm{mm} \mathrm{Hg}$, is recommended. Target BP values in patients with grade 2 and 3 hypertension should be achieved within 3 months of treatment.

\section{Recommendation of a single-pill combination for the initiation of treatment in the majority of patients}

The first step should be two-drug combination therapy, preferably in a single-pill combination. The preferred two-drug combinations include a renin-angiotensin system (RAS) inhibitor with a calcium channel blocker or a diuretic. Monotherapy is recommended only in low-cardiovascular-risk patients with grade 1 hypertension, in very-high-risk patients with high normal BP, in people over 80 years of age and those with frailty syndrome.

\section{Recommendation of three-drug combination in the second step}

If the treatment goal is not achieved with two-drug combination therapy, a three-drug combination based on a RAS inhibitor, a calcium channel blocker (CCB) and a diuretic should be used [6].

\section{Single-pill combinations - an effective way to improve the effectiveness of treatment}

Factors determining insufficient control of blood pressure include factors related to the patient (adherence/compliance, knowledge) and factors related to the doctor (therapeutic inertia).
A common problem that contributes to insufficient BP control worldwide is poor patient adherence to dosing schedules and treatment advice. The degree to which patients' behaviour is consistent with the prescribed treatment is referred to as adherence (or compliance) [7]. Studies assessing patient-doctor relations by means of electronic monitoring have found that $50-60 \%$ of all patients strictly follow medical recommendations (referred as 'adherent' patients), $30-40 \%$ take at least $80 \%$ of the prescribed doses ('partially adherent' patients), while $5-10 \%$ of patients take less than $80 \%$ of the prescribed doses or do not take drugs at all ('non-adherent' patients) $[8,9]$. Adherence to treatment decreases as the number of pills taken daily increases [10].

The introduction of single-pill combinations was helpful in improving antihypertensive effectiveness. It has been shown that adherence to treatment can be significantly improved by reducing the number of pills [11].

It is widely believed that inappropriate doctor's behaviour, including insufficient determination in treatment and not using all therapeutic options, is also an important source of treatment failure. Therapeutic inertia (TI) is defined as failure to intensify treatment despite the fact that therapeutic goals are not achieved. This problem concerns not only BP control, but also other chronic diseases, including diabetes and hyperlipidaemia [12-14].

The above phenomenon is observed especially in patients with relatively slightly elevated blood pressure. The problem of therapeutic inertia was evaluated in an American, retrospective cohort study involving 7253 hypertensive patients treated in 62 medical practices. The TI index was calculated for each patient, with higher values corresponding to higher therapeutic inertia. The main manifestation of TI in his study was the failure to increase the dose of the antihypertensive drug or to add a new drug even though $\mathrm{BP}$ values measured during the clinical visit were above the target range. The study confirmed that the proportion of patients with sufficient blood pressure control is lower in 'real-life' clinical practice than in large clinical trials, and that the incidence of TI is high. Another important observation in this study was the lack of appropriate action, especially in a group of patients at high risk of cardiovascular diseases, defined as coexistence of diabetes, heart failure or other overt cardiovascular diseases, who would benefit the most from appropriate treatment. It was estimated that reducing the TI by $50 \%$ would reduce the average systolic blood pressure by more than $5 \mathrm{~mm} \mathrm{Hg}$ and allow for achieving a BP control rate of $65-70 \%$, which is similar to values reported 
in many clinical trials [15]. Sustained reduction of systolic blood pressure by $5 \mathrm{~mm} \mathrm{Hg}$ for several years reduces the risk of major cardiovascular events by $15-20 \%$ [16].

\section{Problems related to hypertension in metabolic syndrome and diabetes}

Hypertension is 1.5-2 times more prevalent in diabetic patients compared with healthy subjects. The coexistence of these two conditions leads to endothelial dysfunction, vascular damage and acceleration of the atherosclerotic process, and consequently increases the risk of cardiovascular events $[17,18]$. It is estimated that CV risk in this group of patients is 5 times higher than in those without diabetes and without hypertension. Moreover, the presence of hypertension is responsible for a 7.2-fold increase in the risk of death in people with diabetes [19,20].

Metabolic syndrome, often referred to as pre-diabetes, is an independent risk factor of cardiovascular and cerebral events [21].

In the published studies, the risk of cardiovascular events was significantly higher among patients with metabolic syndrome and remained high even after adjusting for age, gender, total cholesterol, creatinine, smoking, left ventricular hypertrophy and 24-hour systolic blood pressure [21, 22 ].

The pathophysiology of hypertension in diabetes and metabolic syndrome is complex. Currently, it is believed that the basic pathophysiological mechanism is endothelial dysfunction manifested by vasoconstriction, increased oxidative stress, vasculitis, prothrombotic state, smooth muscle cell proliferation and impaired repair mechanisms. Along with endothelial damage, the renin-angiotensin-aldosterone system is activated as a key modulator of vascular function $[23,24]$.

Another important pathophysiological mechanism of hypertension in this group of patients is insulin resistance which induces the activation of the sympathetic and the renin-angiotensin-aldosterone systems and the retention of sodium and water [25, 26].

\section{Selection of optimal treatment in the light of the $2018 \mathrm{ESH} / \mathrm{ESC}$ guidelines}

\section{Step one: initiation of antihypertensive therapy}

The choice of antihypertensive therapy in patients with metabolic syndrome and/or diabetes should be made according to current medical standards, selecting drugs with antihypertensive efficacy and cardiovascular protective effect confirmed in EBM trials.

The current 2018 ESH/ESC guidelines indicate the need to lower blood pressure to $130 / 80 \mathrm{~mm} \mathrm{Hg}$ or less (but not lower than 120/70) within 3 months using the first-choice combination of a RAS inhibitor with a calcium channel blocker (CCB) or a diuretic, preferably in the form of a single-pill combination (SPC) [6].

Considering new recommendations and EBM approach, the optimal therapy for the initiation of antihypertensive treatment in patients with diabetes is the combination of perindopril with indapamide. As indicated by evidence from clinical trials, this treatment ensures a significant reduction in $\mathrm{CV}$ risk and a reduction in all-cause and cardiovascular mortality (ADVANCE and ADVANCE-ON) as well as a complete normalization of blood pressure (PICASSO) and metabolic parameters (FORTISSIMO) [27-30].

The use of individualized combination antihypertensive treatment is supported by the fact that the majority of patients with diabetes and hypertension do not achieve blood pressure target values. Analysis of data from the I-SEARCH (International Survey Evaluating Microalbuminuria Routinely by Cardiologists in patients with Hypertension) study showed that among diabetic patients only $19 \%$ of men and in $16 \%$ of women had good blood pressure control, although $93.5 \%$ of patients received antihypertensive drugs [31].

In the ADVANCE (Action in Diabetes and Vascular Disease study: PreterAx and DiamicroN Controlled Evaluation) trial including 11,140 patients with type 2 diabetes, after 4.3 years of treatment with fixed combination of perindopril and indapamide statistically significant reduction compared to placebo was observed in:

- all-cause mortality [relative risk reduction (RRR) $14 \% ; \mathrm{p}=0.025]$;

— cardiovascular mortality (RRR 18\%; $\mathrm{p}=0.027$ );

— total renal events (RRR 21\%; $\mathrm{p}<0.0001$ );

- total coronary events (RRR $14 \% ; \mathrm{p}=0.02)$;

- primary endpoint (total micro- and macroangiopathic events) (RRR 9\%; $\mathrm{p}=0.041)$.

Treatment with perindopril and indapamide was well tolerated; the percentage of adherent patients was $73 \%$ in the experimental group and $74 \%$ in the placebo group [27].

All surviving participants of ADVANCE trial were invited to participate in ADVANCE-ON (Action in Diabetes and Vascular Disease: Preterax and Diamicron Modified Release Controlled Evalu- 
ation Observational Study), both those who were treated with perindopril/indapamide and those receiving placebo. In total, 8494 patients participated in the ADVANCE-ON. The median follow-up after the end of the randomized trial was 5.9 years, and the total duration of the ADVANCE trial and posttrial follow-up was 9.9 years. The analysis of data obtained during nearly 10 years of observation of patients with type 2 diabetes, including the period of the randomized trial and post-trial follow-up, showed a statistically significant reduction in the number of deaths from cardiovascular causes and deaths for any cause, resulting from previous 4.5-year antihypertensive treatment with perindopril and indapamide. The results obtained in the ADVANCE-ON were mainly due to sustained benefits of intensive antihypertensive treatment with a fixed combination of perindopril and indapamide in the ADVANCE study [28]. This proves that an early and effective reduction in blood pressure by fixed combination of perindopril and indapamide translates into long-term clinical benefits.

It is also worth paying attention to the results of the trials assessing the effectiveness of the highest doses of the perindopril/indapamide combination (PICASSO and FORTISSIMO). In an open observational PICASSO (Prevention of Cardiovascular Events in Ischaemic Stroke Patients with High Risk of Cerebral Haemorrhage) trial, the effectiveness of fixed-dose combination of perindopril $10 \mathrm{mg} /$ indapamide $2.5 \mathrm{mg}$ was evaluated in hypertensive patients who failed to achieve BP target values < 140/90 $\mathrm{mm} \mathrm{Hg}$ using standard treatment. Blood pressure significantly decreased from $158.9 \pm 14.3 / 93.0 \pm$ $9.4 \mathrm{~mm} \mathrm{Hg}$ to $131.5 \pm 9.5 / 79.9 \pm 6.2 \mathrm{~mm} \mathrm{Hg}$. Target BP values were achieved by $72.7 \%$ of the 9257 patients included in the final analysis. The $\mathrm{BP}$ reduction was significant regardless of the initial values. Interestingly, significant improvement in the metabolic parameters such as total cholesterol, LDL and HDL cholesterol, triglycerides and blood glucose levels was also observed. The authors conclude that treatment with the perindopril $10 \mathrm{mg} /$ indapamide $2.5 \mathrm{mg}$ combination is effective and safe in patients with poorly controlled blood pressure [29]. Similar conclusions were reached by the authors of the FORTISSIMO (Full-dose Perindopril/Indapamide in the Treatment of Difficult-to-Control Hypertension) trial [30].

This combination is also indicated in patients with metabolic syndrome, which was confirmed by the OPTIMAX study: Real-life Rates of Blood Pressure Normalization With First-Line Therapy (OPTIMAX 2) [32].
The coexistence of abdominal obesity, dyslipidaemia and impaired glucose tolerance is related to a very high risk of developing type 2 diabetes and cardiovascular diseases.

Patients with metabolic syndrome should be treated with medications that have additional beneficial metabolic effects, beyond lowering blood pressure, and delay the development of diabetes [33, 34]. It has been known for a long time that the majority of antihypertensive drugs affects carbohydrate metabolism. Thiazide diuretics, especially when given in high doses, enhance metabolic disorders and increase the risk of diabetes. In a prospective multicentre TROPHY (Treatment in Obese Patients With Hypertension) trial, obese hypertensive patients were randomized to treatment with an ACE inhibitor or hydrochlorothiazide (HCTZ). The therapeutic goal of lowering the diastolic blood pressure below $90 \mathrm{~mm} \mathrm{Hg}$ was obtained by $60 \%$ of patients in the ACE inhibitor group and only $43 \%$ of patients in the HCTZ group. In addition, patients receiving HCTZ showed significant deterioration of the metabolic profile with significantly higher plasma glucose levels and a significant reduction in serum potassium compared to the ACE inhibitor group. This study emphasized that ACE inhibitors are more advantageous and safer in patients with obesity [35]. At the same time, it has been found that HCTZ - not only in monotherapy, but also in combination with other drugs - unfavourably affects the metabolic profile. A subanalysis of the International VErapamil SR Trandalapril STUDy (INVEST) published in 2013, based on pharmacogenetics, demonstrated that the use of HCTZ, even at low doses $(12.5 \mathrm{mg}$ and $25 \mathrm{mg}$ ), in combination with an ACE inhibitor may be in some patients an environmental factor in the development of diabetes. In practice, every 6 months of treatment resulted in a $16 \%$ increase in the risk of developing diabetes [36].

In connection with the above, the guidelines of the Polish Society of Hypertension emphasize that the preferred diuretics should be thiazide-like diuretics (chlorthalidone, indapamide). This recommendation is justified by the evidence for their beneficial effect on $\mathrm{CV}$ risk reduction and a more favourable metabolic profile [5].

Differences in the chemical structure of diuretics are the basis for dividing this group of drugs into thiazides and thiazide-like diuretics (chlorthalidone, indapamide). Different chemical structure translates into different pharmacokinetic properties and potential additional effects. Unique features of indapamide, which are associated with its chemical structure, include its considerable lipophilicity, vaso- 
dilating and antioxidant effects and neutral effect on the lipid profile.

Due to these beneficial pharmacokinetic properties, indapamide is now widely used both in monotherapy and in single-pill combinations with an ACE inhibitor and a CCB. Indapamide is characterized by one of the highest trough-to-peak (T/P) ratios indicating duration of action of more than 24 hours. In a published meta-analysis of 10 randomized trials comparing directly indapamide with HCTZ, indapamide was shown to have significantly higher antihypertensive efficacy than HCTZ [37].

Another published meta-analysis showed that thiazide-like diuretics, including indapamide, statistically significantly reduce the risk of cardiovascular events such as stroke, coronary events and heart failure. For thiazide diuretics, including HCTZ, the reduction of cardiovascular events did not reach statistical significance [38]. The current guidelines of the Polish Society of Hypertension indicate indapamide as the preferred thiazide-like diuretic, and in some groups of patients (those aged over 80 ) it is the only one recommended [5].

\section{Step two: intensification of treatment}

Based on the research, it is estimated that twodrug therapy can effectively control BP in nearly two thirds of patients [39]. For patients who fail to achieve target BP values, the logical option would be to use a combination of three antihypertensive drugs. A three-drug combination enables achieving the target $\mathrm{BP}$ values in more than $80 \%$ of patients [40].

Considering the above, the new 2018 ESH/ESC guidelines recommend that the combination of a RAS inhibitor, a CCB and a diuretic, preferably as a three-drug SPC, should be used for treatment intensification [6].

The Polish Society of Hypertension guidelines highlight the importance of a new group of threedrug combinations and indicate the superiority of single-pill combination of perindopril/indapamide/ amlodipine [5]. This recommendation is supported by EBM studies confirming its effectiveness in reducing CV risk [27, 41, 42]. In addition, this new three-drug combination is characterized by 24 -hour antihypertensive effects of all three drugs which have complementary pharmacokinetic properties in terms of time required to achieve maximum concentration of individual components [43].

Subanalysis of the ADVANCE trial showed significantly more favourable effects in patients with type 2 diabetes receiving single-pill combination of perindopril and indapamide with amlodipine. There was a 2-fold reduction in the risk of death compared to perindopril/indapamide therapy in the entire population of the ADVANCE trial [44].

In the PIANIST (Perindopril-Indapamide plus Amlodipin in high-riSk hyperTensive patients) trial, the efficacy of triple combination of perindopril, indapamide and amlodipine was evaluated in patients with difficult-to-control blood pressure. One third of respondents had ischaemic heart disease or diabetes, and half of the subjects had grade 2 hypertension. The mean office $\mathrm{BP}$ value was $160.5 \pm 13.3 / 93.8$ $\pm 8.7 \mathrm{~mm} \mathrm{Hg}$. The target $\mathrm{BP}$ values were achieved in $72 \%$ of all subjects, in $81 \%$ of patients previously treated with an ACE inhibitor and HCTZ, and in $91 \%$ of patients receiving a sartan and HCTZ prior to the study entry. This analysis of treatment effects in nearly five thousand patients with high or very high cardiovascular risk confirmed the effectiveness of the above triple antihypertensive therapy, regardless of the type of previously used two-drug combination [45]. Another study assessing the combination of perindopril, indapamide, and amlodipine, PETRA (PErindopril based TRiple combination therapy to Achieve 24-hours controlled hypertension), included over 11,000 patients with grade 1,2 and 3 hypertension with associated cardiovascular risk factors and concomitant diseases (dyslipidaemia, diabetes, obesity and coronary heart disease) ). After 3 months of treatment with perindopril, indapamide and amlodipine, systolic blood pressure decreased by 25 $\mathrm{mm} \mathrm{Hg}$, and diastolic blood pressure by $11 \mathrm{~mm} \mathrm{Hg}$. In the context of new recommendations, it should be emphasized that the above combination made it possible to achieve more stringent therapeutic goals of $<130 / 80 \mathrm{~mm} \mathrm{Hg}$ in only 3 months. What's more, $45 \%$ of patients received the lowest dose of the drug $(5 / 1.25 / 5 \mathrm{mg})$ on the last visit [46].

\section{Summary}

Hypertensive patients with diabetes or metabolic syndrome are characterized by high or very high cardiovascular risk. According to the new 2018 ESH/ ESC guidelines, effective prevention of incidents in this group of patients requires achieving target $\mathrm{BP}$ values of systolic blood pressure between 130 and $120 \mathrm{~mm} \mathrm{Hg}$ and diastolic blood pressure between 80 and $70 \mathrm{~mm} \mathrm{Hg}$ within 3 months. The first step of antihypertensive pharmacotherapy should be a twodrug combination of a RAS inhibitor with a diuretic or a CCB, preferably in a SCP. In the second step, a combination of three drugs: a RAS inhibitor, a diuretic and a CCB, preferably in a SPC, should be used in order to intensify the treatment. In accor- 
dance with the current guidelines, respective steps for patients with diabetes and metabolic syndrome should be as follows: the combination of perindopril/ /indapamide as the first step of treatment and threedrug combination of perindopril/indapamide/amlodipine for treatment intensification. This treatment strategy is supported by many EBM trials confirming its effectiveness in achieving target BP values and organ-protective effects.

\section{References}

1. Kearney PM, Whelton M, Reynolds K, et al. Global burden of hypertension: analysis of worldwide data. Lancet. 2005; 365(9455): 217-223, doi: 10.1016/S0140-6736(05)17741-1, indexed in Pubmed: 15652604.

2. Cutler JA, Sorlie PD, Wolz M, et al. Trends in hypertension prevalence, awareness, treatment, and control rates in United States adults between 1988-1994 and 1999-2004. Hypertension. 2008; 52(5): 818-827, doi: 10.1161/HYPERTENSIONAHA.108.113357, indexed in Pubmed: 18852389.

3. Chobonian AV, Bakris GL, Black HR, et al. The Seventh Report of the Joint National Committee on Prevention, Detection, Evaluation, and Treatment of High Blood Pressure: the JNC7 report. JAMA. 2003; 289(19): 2560-2572 [Errata, JAMA 2003; 290: 197], doi: 10.1001/jama.289.19.2560, indexed in Pubmed: 12748199.

4. Mancia G, Fagard R, Narkiewicz K, et al. 2013 ESH/ESC guidelines for the management of arterial hypertension: the Task Force for the Management of Arterial Hypertension of the European Society of Hypertension (ESH) and of the European Society of Cardiology (ESC). Eur Heart J. 2013; 34(28): 2159-2219, doi: 10.1093/ eurheartj/eht151, indexed in Pubmed: 23771844.

5. Tykarski A, Narkiewicz K, Gaciong Z, et al. Guidelines for the Management of Hypertension. Arterial Hypertension. 2015; 19(2): 53-83, doi: 10.5603/ah.2015.0010.

6. Williams B, Mancia G, Spiering W, et al. ESC Scientific Document Group . 2018 ESC/ESH Guidelines for the management of arterial hypertension. Eur Heart J. 2018; 39(33): 3021-3104, doi: 10.1093/ eurheartj/ehy339, indexed in Pubmed: 30165516.

7. Burnier M. Drug adherence in hypertension. Pharmacol Res. 2017; 125: 142-149, doi: 10.1016/j.phrs.2017.08.015.

8. Xu T, Yu X, Ou S, et al. Adherence to antihypertensive medications and stroke risk: a dose-response meta-analysis. J Am Heart Assoc. 2017; 6(7), doi: 10.1161/JAHA.117.006371, indexed in Pubmed: 28743788 .

9. Krousel-Wood M, Thomas S, Muntner P, et al. Medication adherence: a key factor in achieving blood pressure control and good clinical outcomes in hypertensive patients. Curr Opin Cardiol. 2004; 19(4): 357-362, indexed in Pubmed: 15218396.

10. Mengden T, Binswanger B, Spühler T, et al. The use of selfmeasured blood pressure determinations in assessing dynamics of drug compliance in a study with amlodipine once a day, morning versus evening. J Hypertens. 1993; 11(12): 1403-1411, indexed in Pubmed: 8133022.

11. Eisen SA, Miller DK, Woodward RS, et al. The effect of prescribed daily dose frequency on patient medication compliance. Arch Intern Med. 1990; 150(9): 1881-1884, indexed in Pubmed: 2102668.

12. Oliveria SA, Lapuerta P, McCarthy BD, et al. Physician-related barriers to the effective management of uncontrolled hypertension. Arch Intern Med. 2002; 162(4): 413-420, indexed in Pubmed: 11863473

13. Ziemer DC, Doyle JP, Barnes CS, et al. Clinical inertia. Ann Intern Med. 2001; 135(9): 825-834, indexed in Pubmed: 11694107.

14. AndradeSE, GurwitzJH, FieldTS, et al. Hypertension management: the care gap between clinical guidelines and clinical practice. Am J Manag Care. 2004; 10(7 Pt 2): 481-486, indexed in Pubmed: 15298234.
15. Okonofua EC, Simpson KN, Jesri A, et al. Therapeutic inertia is an impediment to achieving the Healthy People 2010 blood pressure control goals. Hypertension. 2006; 47(3): 345-351, doi: 10.1161/01.HYP.0000200702.76436.4b, indexed in Pubmed: 16432045.

16. Reach G, Pechtner V, Gentilella R, et al. Clinical inertia and its impact on treatment intensification in people with type 2 diabetes mellitus. Diabetes Metab. 2017; 43(6): 501-511, doi: 10.1016/j. diabet.2017.06.003, indexed in Pubmed: 28754263.

17. Hu G, Jousilahti P, Tuomilehto J. Joint effects of history of hypertension at baseline and type 2 diabetes at baseline and during follow-up on the risk of coronary heart disease. Eur Heart J. 2007; 28(24): 3059-3066, doi: 10.1093/eurheartj/ehm501, indexed in Pubmed: 17981826.

18. Menke A, Casagrande S, Geiss L, et al. Prevalence of and Trends in Diabetes Among Adults in the United States, 1988-2012. JAMA. 2015; 314(10): 1021-1029, doi: 10.1001/jama.2015.10029, indexed in Pubmed: 26348752.

19. Tatsumi Y, Ohkubo T. Hypertension with diabetes mellitus: significance from an epidemiological perspective for Japanese. Hypertens Res. 2017; 40(9): 795-806, doi: 10.1038/hr.2017.67, indexed in Pubmed: 28701739 .

20. Stratton IM, Cull CA, Adler AI, et al. Additive effects of glycaemia and blood pressure exposure on risk of complications in type 2 diabetes: a prospective observational study (UKPDS 75). Diabetologia. 2006; 49(8): 1761-1769, doi: 10.1007/s00125-006-0297-1, indexed in Pubmed: 16736131.

21. Schillaci G, Pirro M, Vaudo G, et al. Prognostic value of the metabolic syndrome in essential hypertension. J Am Coll Cardiol. 2004; 43(10): 1817-1822, doi: 10.1016/j.jacc.2003.12.049, indexed in Pubmed: 15145106

22. Mancia G, Bombelli M, Corrao G, et al. Metabolic syndrome in the Pressioni Arteriose Monitorate E Loro Associazioni (PAMELA) study: daily life blood pressure, cardiac damage, and prognosis. Hypertension. 2007; 49(1): 40-47, doi: 10.1161/01. HYP.0000251933.22091.24, indexed in Pubmed: 17130308.

23. Incalza MA, D'Oria R, Natalicchio A, et al. Oxidative stress and reactive oxygen species in endothelial dysfunction associated with cardiovascular and metabolic diseases. Vascul Pharmacol. 2018; 100: 1-19, doi: 10.1016/j.vph.2017.05.005, indexed in Pubmed: 28579545.

24. Haas AV, McDonnell ME. Pathogenesis of Cardiovascular Disease in Diabetes. Endocrinol Metab Clin North Am. 2018; 47(1): 51-63, doi: 10.1016/j.ecl.2017.10.010, indexed in Pubmed: 29407056.

25. Safar M. Arterial stiffness as a risk factor for clinical hypertension. Nat Rev Cardiol. 2017; 15(2): 97-105, doi: 10.1038/nrcardio.2017.155.

26. Libianto R, Batu D, MacIsaac RJ, et al. Pathophysiological Links Between Diabetes and Blood Pressure. Can J Cardiol. 2018; 34(5): 585-594, doi: 10.1016/j.cjca.2018.01.010, indexed in Pubmed: 29731021.

27. Patel A. Effects of a fixed combination of perindopril and indapamide on macrovascular and microvascular outcomes in patients with type 2 diabetes mellitus (the ADVANCE trial): a randomised controlled trial. Lancet. 2007; 370(9590): 829-840, doi: 10.1016/s01406736(07)61303-8.

28. Zoungas S, Chalmers J, Neal B, et al. ADVANCE-ON Collaborative Group. Follow-up of blood-pressure lowering and glucose control in type 2 diabetes. N Engl J Med. 2014; 371(15): 1392-1406, doi: 10.1056/NEJMoa1407963, indexed in Pubmed: 25234206.

29. Farsang C. Picasso Investigators. Blood pressure and metabolic efficacy of fixed-dose combination of perindopril and indapamide in everyday practice. Blood Press. 2013; 22 Suppl 1: 3-10, doi: 10.3109/08037051.2012.740787, indexed in Pubmed: 23163322.

30. Karpov YA. FORTISSIMO physicians. Full-dose Perindopril/Indapamide in the Treatment of Difficult-to-Control Hypertension: The FORTISSIMO Study. Clin Drug Investig. 2017; 37(2): 207-217, doi: 10.1007/s40261-016-0479-7, indexed in Pubmed: 27878562.

31. Thoenes M, Neuberger HR, Volpe M, et al. Antihypertensive drug therapy and blood pressure control in men and women: an interna- 
tional perspective. J Hum Hypertens. 2010; 24(5): 336-344, doi: 10.1038/jhh.2009.76, indexed in Pubmed: 19798089.

32. Mourad JJ, Lameira D, Guillausseau PJ. Blood pressure normalization by fixed perindopril/indapamide combination in hypertensive patients with or without associate metabolic syndrome: results of the OPTIMAX 2 study. Vasc Health Risk Manag. 2008; 4(2): 443-451, indexed in Pubmed: 18561520.

33. Isomaa B, Almgren P, Tuomi T, et al. Cardiovascular morbidity and mortality associated with the metabolic syndrome. Diabetes Care. 2001; 24(4): 683-689, indexed in Pubmed: 11315831.

34. Klein BEK, Klein R, Lee KE. Components of the metabolic syndrome and risk of cardiovascular disease and diabetes in Beaver Dam. Diabetes Care. 2002; 25(10): 1790-1794, indexed in Pubmed: 12351479

35. Reisin E, Weir MR, Falkner B, et al. Lisinopril versus hydrochlorothiazide in obese hypertensive patients: a multicenter placebo-controlled trial. Treatment in Obese Patients With Hypertension (TROPHY) Study Group. Hypertension. 1997; 30(1 Pt 1): 140-145, indexed in Pubmed: 9231834.

36. Karnes JH, Gong Y, Pacanowski MA, et al. Impact of TCF7L2 single nucleotide polymorphisms on hydrochlorothiazide-induced diabetes. Pharmacogenet Genomics. 2013; 23(12): 697-705, doi: $10.1097 /$ FPC.0000000000000012, indexed in Pubmed: 24128935.

37. Roush GC, Sica DA. Diuretics for hypertension: a review and update. Am J Hypertens. 2016; 29(10): 1130-1137, doi: 10.1093/ajh/ hpw030, indexed in Pubmed: 27048970.

38. Chen P, Chaugai S, Zhao F, et al. Cardioprotective effect of thiazidelike diuretics: a meta-analysis. Am J Hypertens. 2015; 28(12): 14531463, doi: 10.1093/ajh/hpv050, indexed in Pubmed: 25926533.

39. Wald DS, Law M, Morris JK, et al. Combination therapy versus monotherapy in reducing blood pressure: meta-analysis on 11,000 participants from 42 trials. Am J Med. 2009; 122(3): 290-300, doi: 10.1016/j.amjmed.2008.09.038, indexed in Pubmed: 19272490.

40. Weir MR, Hsueh WA, Nesbitt SD, et al. A titrate-to-goal study of switching patients uncontrolled on antihypertensive monotherapy to fixed-dose combinations of amlodipine and olmesartan medoxomil \pm hydrochlorothiazide. J Clin Hypertens (Greenwich). 2011; 13(6): 404-412, doi: 10.1111/j.1751-7176.2011.00437.x, indexed in Pubmed: 21649839.

41. Beckett NS, Peters R, Fletcher AE, et al. HYVET Study Group. Treatment of hypertension in patients 80 years of age or older. $\mathrm{N}$ Engl J Med. 2008;358(18): 1887-1898, doi: 10.1056/NEJMoa0801369, indexed in Pubmed: 18378519.

42. Randomised trial of a perindopril-based blood-pressure-lowering regimen among 6105 individuals with previous stroke or transient ischaemic attack. The Lancet. 2001; 358(9287): 1033-1041, doi: 10.1016/s0140-6736(01)06178-5.

43. Tykarski A, Widecka K, Narkiewicz K, et al. Leki złożone w terapii nadciśnienia tętniczego w Polsce. Stanowisko Ekspertów Polskiego Towarzystwa Nadciśnienia Tętniczego oraz Sekcji Farmakoterapii Sercowo-Naczyniowej Polskiego Towarzystwa Kardiologicznego. Arterial Hypertension. 2017; 21(3): 105-115, doi: 10.5603/ ah.2017.0014.

44. Chalmers J, Arima H, Woodward M, et al. Effects of combination of perindopril, indapamide, and calcium channel blockers in patients with type 2 diabetes mellitus: results from the Action In Diabetes and Vascular Disease: Preterax and Diamicron Controlled Evaluation (ADVANCE) trial. Hypertension. 2014; 63(2): 259-264, doi: 10.1161/HYPERTENSIONAHA.113.02252, indexed in Pubmed: 24324048

45. Tóth K. PIANIST Investigators. Antihypertensive efficacy of triple combination perindopril/indapamide plus amlodipine in high-risk hypertensives: results of the PIANIST study (Perindopril-Indapamide plus AmlodipiNe in high rISk hyperTensive patients). Am J Cardiovasc Drugs. 2014; 14(2): 137-145, doi: 10.1007/s40256-0140067-2, indexed in Pubmed: 24590580.

46. Ábrahám G, Dézsi CA. The antihypertensive efficacy of the triple fixed combination of perindopril, indapamide, and amlodipine: the results of the PETRA study. Adv Ther. 2017; 34(7): 1753-1763, doi: 10.1007/s12325-017-0572-1, indexed in Pubmed: 28646394. 